\title{
Repetitive visual stimulation enhances recovery from severe amblyopia
}

\author{
Karen L. Montey, ${ }^{1}$ Nicolette C. Eaton, ${ }^{1}$ and Elizabeth M. Quinlan ${ }^{1,2,3}$ \\ ${ }^{1}$ Department of Biology, University of Maryland, College Park, Maryland 20742, USA; ${ }^{2}$ Neuroscience and Cognitive Science Program, \\ University of Maryland, College Park, Maryland 20742, USA
}

\begin{abstract}
Severe amblyopia, characterized by a significant reduction in visual acuity through the affected eye, is highly resistant to reversal in adulthood. We have previously shown that synaptic plasticity can be reactivated in the adult rat visual cortex by dark exposure, and the reactivated plasticity can be harnessed to promote the recovery from severe amblyopia. Here we show that deprived-eye visually evoked responses are rapidly strengthened in dark-exposed amblyopes by passive viewing of repetitive visual stimuli. Surprisingly, passive visual stimulation rapidly enhanced visually evoked responses to novel stimuli and enhanced the recovery from severe amblyopia driven by performance of active visual discriminations. Thus a series of simple, noninvasive manipulations of visual experience can be used in combination to significantly guide the recovery of visual response strength, selectivity, and spatial acuity in adult amblyopes.
\end{abstract}

Monocular deprivation initiated during a postnatal critical period reduces visual acuity in the pathway served by the deprived eye. The age at onset and the duration of the monocular deprivation determine the severity of the visual deficit, as well as the potential for recovery. For example, the visual deficits induced by brief monocular lid suture at the peak of the critical period reverse spontaneously following removal of the occlusion (Liao et al. 2004).

In contrast, the presence of a unilateral congenital cataract at birth, or monocular lid suture at eye opening, induces severe amblyopia that is particularly resistant to reversal later in postnatal life (Wiesel and Hubel 1965; Mitchell and MacKinnon 2002; He et al. 2007). The significant reduction in cortical plasticity that accompanies aging contributes to the constraint on the experiencedependent recovery from amblyopia in adulthood. However, this impediment can be removed by dark exposure, which reactivates synaptic plasticity in the adult visual cortex (He et al. 2007; Montey and Quinlan 2011). In addition, visual stimulation of a chronically deprived eye continues to evoke residual cortical responses (Montey and Quinlan 2011). The residual connectivity between the chronically deprived eye and the primary visual cortex provides a potential anatomical substrate for experiencedependent strengthening of the deprived-eye pathway.

An emerging body of evidence demonstrates that plasticity of visual responses can be induced by repetitive visual experience. For example, repeated performance in a visual texture discrimination task improves visual perception and lowers discrimination thresholds in humans (Karni and Sagi 1993). Work in other species demonstrates that repetitive visual exercises, such as orientation detection or spatial frequency discrimination, narrow the tuning of activated cortical neurons and increase the strength of synapses in the primary visual cortex (Schoups et al. 2001; Sale et al. 2011). Plasticity of visual responses can also be induced by passive visual stimulation. For example, passive viewing of reversing highcontrast checkerboards increases the amplitude of the visually evoked component of the EEG in humans (Teyler et al. 2005). Similarly, passive viewing of reversing high-contrast gratings increases the amplitude of the visually evoked potential (VEP) in the primary visual cortex of binocular mice (Cooke and Bear

\section{${ }^{3}$ Corresponding author}

E-mail equinlan@umd.edu

Article is online at http://www.learnmem.org/cgi/doi/10.1101/Im.030361.113.
2010). Importantly, such passive visual experience can regulate stimulus discrimination thresholds (Beste et al. 2011). However, the enhancement of visual responses driven by passive or active visual stimulation is typically restricted to the features of the familiar visual stimulus, and rarely transfers to novel visual stimuli.

Importantly, the ability to regulate visual responses by repetitive visual experience is retained by the amblyopic visual system (Levi and Li 2009). Indeed, active visual discrimination improves perception and lowers discrimination thresholds in amblyopic humans, cats, and rats (Giffin and Mitchell 1978; Iny et al. 2006; Zhou et al. 2006). Similarly, passive viewing of repetitive visual stimuli improves spatial acuity in amblyopic children (Willshaw et al. 1980; Lennerstrand et al. 1981). The use of repetitive visual experience clearly holds promise for improving vision in amblyopes. However, the improvements in spatial acuity reported to date are modest, and are likely constrained by the reduction in cortical plasticity that occurs over development. Here we show that the reactivation of synaptic plasticity in the adult visual cortex by dark exposure significantly enhances the cortical response to repetitive visual stimulation. Importantly, passive visual stimulation significantly enhanced the recovery of spatial acuity driven by active visual discrimination in dark-exposed amblyopes.

\section{Results}

Chronic monocular deprivation induces severe amblyopia The consequence of chronic monocular deprivation (from eye opening at postnatal day 14 to adulthood at postnatal day 120) on visually evoked neuronal activity was examined with single unit recordings from the deprived visual cortex (binocular primary visual cortex contralateral to the deprived eye). Polar plots of the activity of a representative layer IV neuron demonstrate that chronic monocular deprivation decreases the strength and orientation selectivity of spiking activity evoked by visual stimulation of the deprived eye relative to the nondeprived eye (Fig. 1B). Post-stimulus time histograms reveal that the nondeprived eye retains orientation selectivity (Fig. 1C). In contrast, visual stimulation of the chronically deprived eye evoked a similar response to visual stimuli of all orientations (Fig. 1D). The cumulative distributions of all visually evoked activity confirms a significant decrease in the strength (evoked activity [spikes/sec]: deprived eye vs. 
A

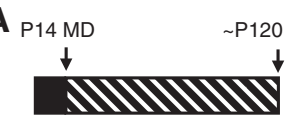

B

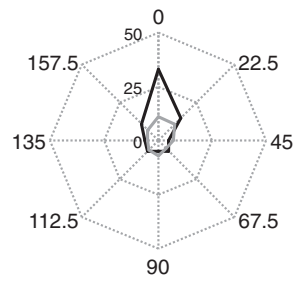

Orientation (degrees)

E

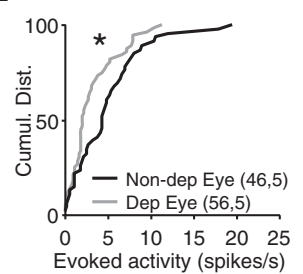

C

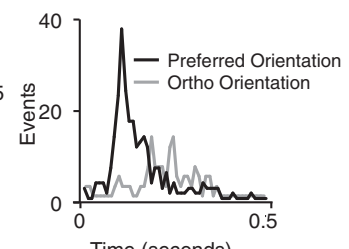

$\mathbf{F}$

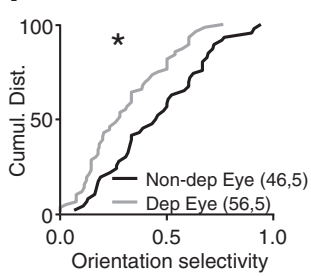

D Dep Eye

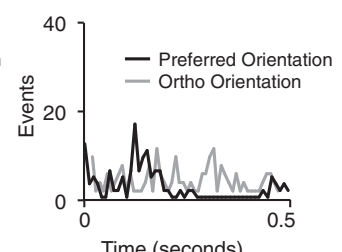

G

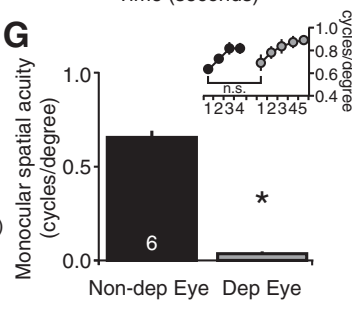

Figure 1. Chronic monocular deprivation induces severe amblyopia. (A) Experimental timeline: chronic monocular deprivation (MD) was initiated at eye opening (postnatal day 14 [P14]) and maintained into adulthood (postnatal day 120 [P120]). (B) Polar plots of a representative layer IV neuron reveal a decrease in the strength and orientation selectivity of spiking output evoked by visual stimulation of the deprived eye (Dep Eye, gray) relative to the nondeprived eye (non-Dep Eye [black] 25 cycles of 0.05 cycles/degree, $100 \%$ contrast full-field vertical gratings reversing at $0.5 \mathrm{~Hz}$ ). (C) Post-stimulus time histograms of spiking output from a representative layer IV neuron evoked by visual stimuli of preferred (black) and orthogonal (gray) orientations presented to the nondeprived eye. (D) Post-stimulus time histograms of spiking output from a representative layer IV neuron evoked by visual stimuli of preferred (black) and orthogonal (gray) orientations presented to the deprived eye. (E) The cumulative distributions of single unit activity reveal a significant decrease in neuronal spiking rates evoked by stimulation of the deprived eye (gray) relative to the nondeprived eye (black) (KS test, $P=0.004$, $n=$ units, subjects). $(F)$ The cumulative distributions of single unit activity reveal significantly less orientation tuning in the neuronal spiking evoked by stimulation of the deprived eye (gray) relative to the nondeprived eye (black). (G) Chronic monocular deprivation induced a significant decrease in the spatial acuity of the deprived eye (gray) (paired $t$-test, $P<0.001$ ); (inset) nondeprived eye spatial acuity assessed with a vertical (trained) stimulus (black) and a novel stimulus rotated $45^{\circ}$ from vertical (gray) reveals a significant decrease in spatial acuity (paired $t$-test, $P<0.05$ ) after a change in stimulus orientation.

nondeprived eye, Kolmogorov-Smirnov [KS] test, $P=0.004$ ) (Fig. $1 \mathrm{E})$ and orientation tuning of deprived eye visually evoked responses (orientation selectivity $=($ response evoked by visual stimulus in preferred orientation - orthogonal orientation) $/($ pref + ortho): deprived eye vs. nondeprived eye, KS test, $P=0.044)$ (Fig. 1F).

The significant reduction in visually evoked response strength and selectivity induced by chronic monocular deprivation is predicted to significantly reduce the acuity of vision through the deprived eye. To confirm this prediction we used a two-alternative, forced choice spatial frequency discrimination task to assess spatial acuity (see Materials and Methods; Prusky et al. 2000). Subjects discriminate between high-contrast vertical gratings of increasing spatial frequency and equal luminance gray screen with their nondeprived eye until performance plateaus (Fig. 1G, inset). We found that subsequent rotation of the visual stimulus to a novel (horizontal) orientation decreases performance in the task. Therefore, the highest spatial frequency that transfers to the novel visual stimulus orientation is reported as baseline spatial acuity (Fig. 1G). Following determination of nondeprived eye spatial acuity, subjects are reverse sutured, and the paradigm is repeated to determine the spatial acuity of the deprived eye, which is significantly reduced (cycles/degree, Dep Eye, $0.036 \pm 0.011$; non-Dep Eye, $0.652+0.038, n=6$; paired $t$-test, $P<0.001$ ) (Fig. 1G). Together this demonstrates that chronic monocular deprivation induces severe amblyopia, characterized

by a significant decrease in deprived eye visual response strength, selectivity, and spatial acuity.

\section{Dark exposure enables response enhancement to repetitive visual stimulation in amblyopic adults} Severe amblyopia is particularly resistant to reversal in adulthood. However, we hypothesized that reactivation of synaptic plasticity in the adult visual cortex by dark exposure would enable experiencedependent plasticity of visual responses. We adopted a visual stimulation protocol recently shown to induce a slow, stimulus-selective enhancement of visually evoked responses in binocular mice (Frenkel et al. 2006; Cooke and Bear 2010). In our experiments, repetitive visual stimulation (100 cycles of 0.05 cycles/degree, $100 \%$ contrast full-field vertical gratings reversing at $0.5 \mathrm{~Hz}$ ) was presented monocularly to subjects that were head-restrained and anesthetized ( $\sim 2 \%$ isoflurane). Visual stimulation of the chronically deprived eye induced a rapid enhancement of the amplitude of layer IV VEPs in dark-exposed amblyopes (normalized VEP amplitudes, average \pm SEM, 120 min post $1.47 \pm 0.13,240 \mathrm{~min}$ post $1.84 \pm 0.13, n=7$, one-way ANOVA, $F_{(2,18)}=18.3033, P<0.001 ; P<0.05$ vs. baseline, Tukey-Kramer post hoc) (Fig. 2B). In contrast, visual stimulation of the chronically deprived eye did not induce a rapid enhancement of VEP amplitudes in amblyopes that were not dark exposed (normalized VEP amplitudes, average \pm SEM, 120 min post $0.88 \pm 0.08,240 \mathrm{~min}$ post $0.93 \pm 0.08, n=5$, one-way ANOVA, $F_{(2,12)}=0.4602, P=$ 0.4602 vs. baseline) (Fig. 2C). The rapid response to repetitive visual stimulation was not due to lower initial VEP amplitudes, as this was unchanged by dark exposure (VEP amplitude $[\mu \mathrm{V}]$ average \pm SEM, no dark exposure, $122.2 \pm 13.56, n=5$; dark exposure, $92.0 \pm$ $17.35, n=7$; unpaired $t$-test, $P=0.231$ [not shown]).

The increase in visually evoked response strength induced by repetitive visual stimulation is typically highly input-specific. Surprisingly, however, the response to repetitive passive visual stimulation in dark-exposed amblyopes was not limited to familiar visual stimuli. Indeed, following passive viewing of 0.05 cycles/ degree vertical gratings, novel visual stimuli of a range of spatial frequencies evoked VEPs with enhanced amplitudes (Fig. 2D). As a result, the extrapolation to $0 \mathrm{mV}$ of the linear regression of spatial frequency vs. VEP amplitude was significantly higher following passive visual stimulation in dark-exposed amblyopes compared to amblyopes without dark exposure (average \pm SEM, Dep Eye + Stim, $0.35 \pm 0.05$; Dep Eye DE + Stim, $0.73 \pm 0.06$; unpaired $t$-test, $P<0.001)$. Similarly, novel visual stimuli rotated up to $45^{\circ}$ from the familiar orientation evoked VEPs with enhanced amplitudes only in dark-exposed amblyopes (VEP amplitudes average \pm SEM, novel/familiar orientation, degrees from familiar, $0=1.84 \pm$ $0.12, \quad 22.5=1.77 \pm 0.14, \quad 45=1.81 \pm 0.16, \quad 67.5=1.60 \pm 0.07$, $90=1.52 \pm 0.16 ;$ non-dark exposed, $0=1.15 \pm 0.28, \quad 22.5=$ $1.21 \pm 0.22, \quad 45=1.18 \pm 0.21, \quad 67.5=1.15 \pm 0.26, \quad 90=1.13 \pm$ 0.21 ; repeated measures ANOVA, $F_{(1,10)}=6.544, \quad P=0.028$ ) 
A
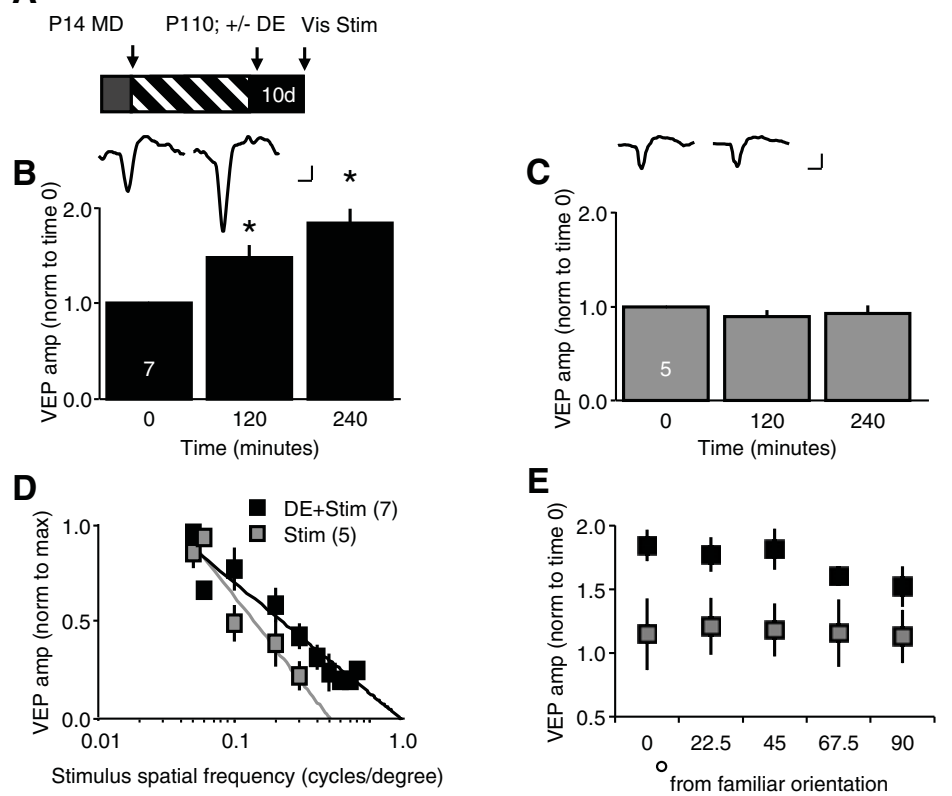

E
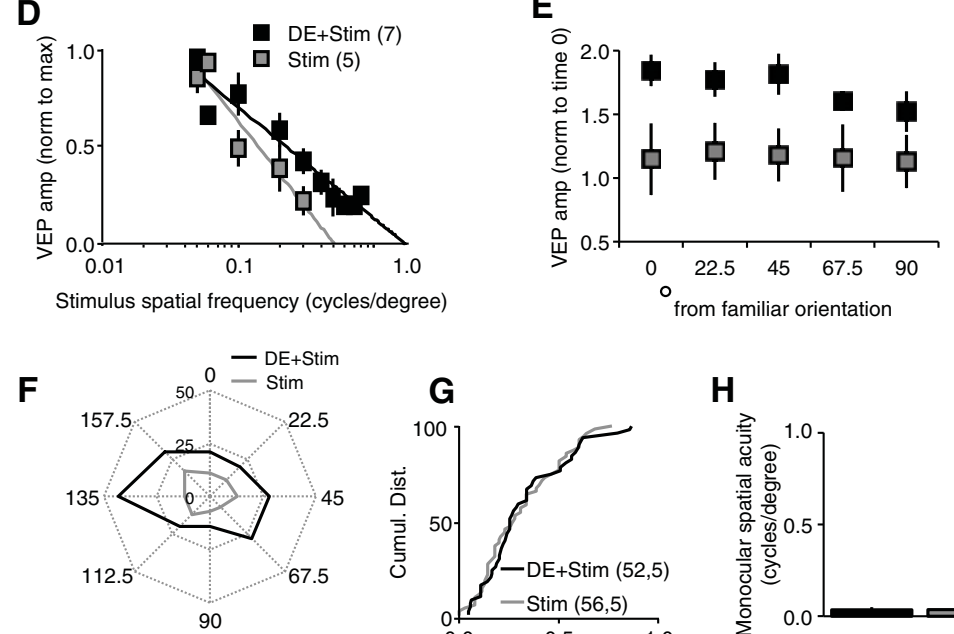

Orientation (degrees)

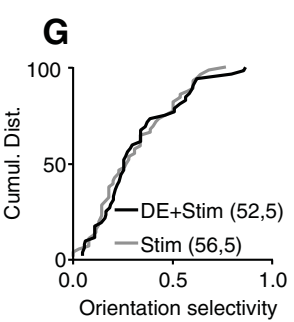

H

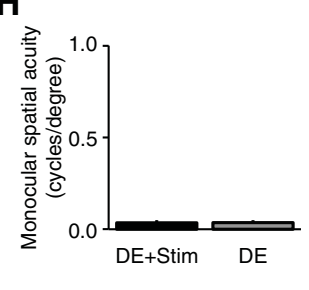

Figure 2. Passive viewing of repetitive visual stimuli induces a rapid, noninput specific enhancement of visual responses in dark-exposed amblyopes. $(A)$ Experimental timeline: following chronic monocular deprivation (MD; from P14 to P110), one cohort received $10 \mathrm{~d}$ of dark exposure (DE). Subsequently, all subjects received reverse deprivation, and passively viewed repetitive visual stimuli (100 cycles of 0.05 cycles/degree, $100 \%$ contrast full-field vertical gratings reversing at $0.5 \mathrm{~Hz}$ ) with the previously deprived eye (Dep Eye). (B) Passive visual stimulation (Stim) induced a rapid enhancement of layer IV VEP amplitudes in dark-exposed amblyopes (average \pm SEM, one-way ANOVA, $F_{(2,18)}=18.3033, P<$ $0.0001,\left[{ }^{*}\right] P<0.05$ vs. baseline, Tukey Kramer HSD post hoc); (inset) representative deprived eye VEPs (Dep Eye) at time 0 (baseline) and 240 min post passive visual stimulation. (C) Passive visual stimulation (Stim) did not induce a rapid enhancement of layer IV VEP amplitudes in amblyopes that did not receive dark exposure (average \pm SEM); (inset) representative deprived eye VEPs (Dep Eye) at time 0 (baseline) and 240 min post passive visual stimulation. (D) Visual stimuli with novel spatial frequencies evoked enhanced VEPs in dark-exposed amblyopes that received passive visual stimulation (black) (average amplitude [norm to max] \pm SEM). (E) Visual stimuli with novel orientations evoked enhanced VEPs in dark-exposed amblyopes that received passive visual stimulation (black) (average \pm SEM, repeated measures ANOVA $F_{(1,10)}=6.544, P=0.028$. $(F)$ Polar plots of representative layer IV neurons demonstrate that passive repetitive visual stimulation (Stim [black]) increased the strength of visually evoked spiking across all orientations. (G) No improvement in orientation selectivity of visually evoked spiking output following passive repetitive visual stimulation in dark-exposed (DE) amblyopes (black) versus control amblyopes without dark exposure (gray; KS test, $P=0.908 ; n=$ units, subjects). (H) Passive visual stimulation (Stim) did not improve spatial acuity of the deprived eye of dark exposed (DE) amblyopes (average monocular spatial acuity \pm SEM, DE + Stim [black] $0.035 \pm 0.013$, $n=7$; DE [gray], $0.036 \pm 0.011, n=6$; $t$-test, $P=0.955)$. Scale bars: $50 \mu \mathrm{V} ; 50$ msec.

(Fig. 2E). Thus the response to repetitive visual stimulation in darkexposed amblyopes was rapid and noninput specific.

We next asked if the repetitive visual stimulation increased the strength or selectivity of visually evoked single unit activity. Polar plots of a representative layer IV neuron demonstrate that visual stimulation increases the strength of response to visual stimuli of all orientations in a dark-exposed amblyope (Fig. 2F). The cumulative distributions of all visually evoked activity confirms a significant increase in the strength of deprived eye responses following passive visual stimulation (DE 52,5, KS test, $P=0.020$ ), but did not change spontaneous neuronal spike rate (KS test, $P=0.173$ ). Accordingly, passive repetitive visual stimulation did not induce a recovery orientation selectivity of visually evoked spiking output in dark-exposed amblyopes (KS test, $P=0.908$ vs. amblyopes without dark exposure) (Fig. 2G). Similarly, passive repetitive visual stimulation did not increase monocular spatial acuity assessed behaviorally (deprived eye spatial acuity, cycles/degree, visual stimulation, $0.035 \pm 0.013, n=7$; no stimulation, $0.036 \pm 0.011, n=6$; $t$-test, $P=$ 0.955) (Fig. 2H). Thus, passive visual stimulation induced widespread changes in the strength of visual responses that were rapid and noninput specific in dark-exposed amblyopes, without regulating the orientation selectivity or spatial acuity of the deprived eye.

We proposed that dark exposure enabled the rapid enhancement of VEP amplitudes by passive visual stimulation in amblyopic adults. To ask if similar plasticity could be engaged in nonamblyopic subjects, we examined the effect of $10 \mathrm{~d}$ of dark exposure on the response to repetitive passive visual stimulation in binocular adults. Surprisingly, dark exposure did not enable a rapid response to repetitive visual stimulation in binocular subjects (VEP amplitude normalized to baseline [time 0], average \pm SEM, dark exposure, $120 \mathrm{~min}$ post $1.24 \pm 0.27,240$ min post $1.30 \pm 0.17, n=5$; no dark exposure, $120 \mathrm{~min}$ post $1.02 \pm 0.13,240$ min post $1.14 \pm 0.10, n=5$ ) (Fig. 3B,C). Similarly, visual stimulation did not induce an enhancement in VEP amplitudes in response to visual stimuli of novel spatial frequencies (average \pm SEM, dark exposure, $1.04 \pm 0.06$; no dark exposure, $1.09 \pm 0.10$; unpaired $t$-test, $P<$ 0.001 ) or orientations (VEP amplitudes average \pm SEM, novel/familiar orientation, degrees from familiar, dark exposed, $0=1.28 \pm 0.18,22.5=1.26 \pm 0.23,45=$ $1.34 \pm 0.37, \quad 67.5=1.16 \pm 0.19, \quad 90=$ $1.28 \pm 0.26$; no dark exposure, $0=1.14$ $\pm 0.10,22.5=1.06 \pm 0.10,45=1.10 \pm$ $0.11, \quad 67.5=1.04 \pm 0.07, \quad 90=1.07 \pm$ 0.06) (Fig. 3D,E). Again, initial VEP amplitudes were unchanged by dark exposure alone $(\mu \mathrm{V}$, average \pm SEM control, $225.2 \pm 36.91, n=5 ; \mathrm{DE}, 217.8 \pm 37.67$, $n=5$ ). This demonstrates that both chronic monocular deprivation and dark exposure are necessary to enable the rapid, noninput specific enhancement of VEP amplitudes by repetitive passive visual stimulation.

\section{Visual stimulation optimizes recovery from severe amblyopia}

Our previous attempts to use active visual discrimination to drive recovery from severe amblyopia may have had modest success 
A
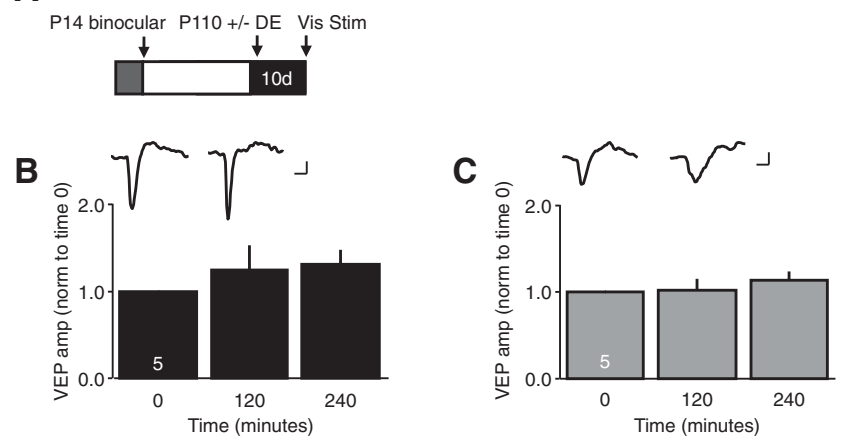

D

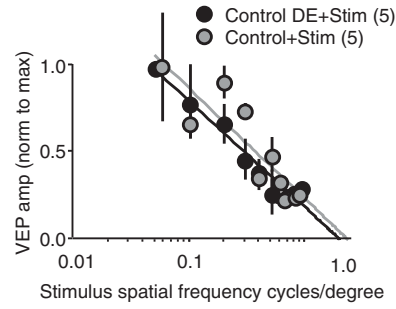

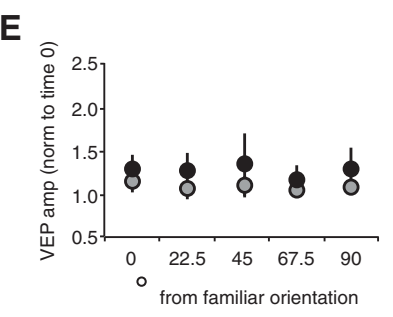

Figure 3. Passive viewing of repetitive visual stimuli does not induce a rapid enhancement of visual responses in dark-exposed binocular subjects. (A) Experimental timeline: following normal binocular visual experience (12-h light/12-h dark/day, from P14 to P110), one cohort received $10 \mathrm{~d}$ of dark exposure (DE). Subsequently, all subjects passively viewed repetitive visual stimuli (Stim, 100 cycles of 0.05 cycles/degree, $100 \%$ contrast full-field vertical gratings reversing at $0.5 \mathrm{~Hz}$ ) with their dominant, contralateral eye. (B) Passive visual stimulation (Stim) did not induce a rapid enhancement of layer IV VEPs in dark-exposed binocular subjects (average \pm SEM); (inset) representative contralateral eye VEPs at time 0 (baseline) and 240 min post visual stimulation. (C) Passive visual stimulation did not induce a rapid enhancement of layer IV VEPs in binocular subjects that did not receive dark exposure (average \pm SEM); (inset) representative contralateral eye VEPs at time 0 (baseline) and $240 \mathrm{~min}$ post visual stimulation. $(D)$ Visual stimuli with novel spatial frequencies did not evoke VEPs with enhanced amplitudes in binocular subjects that received passive visual stimulation (average amplitude [norm to max] \pm SEM). (E) Visual stimuli with novel orientations did not evoke VEPs with enhanced amplitudes in binocular subjects that received passive visual stimulation (average \pm SEM). Scale bars: $50 \mu \mathrm{V} ; 50 \mathrm{msec}$.

because the weakened synapses serving the deprived eye do not strongly influence neuronal spiking. However, we predicted that strengthening synapses with repetitive visual stimulation would improve the recovery of vision by subsequent active visual discriminations. Therefore, we examined the effect of repetitive passive visual stimulation on the recovery from severe amblyopia driven by performance in an active visual discrimination task. Chronically deprived subjects received $10 \mathrm{~d}$ of dark exposure, followed by reverse deprivation (open chronically deprived eye, close nondeprived eye), and repetitively performed the spatial frequency discrimination task with the previously deprived eye $(\sim 25$ trials/d, three times a week for 35 sessions). Two hours prior to performing the task, one cohort received passive visual stimulation (100 cycles of 0.05 cycles/degree, $100 \%$ contrast full-field vertical gratings reversing at $0.5 \mathrm{~Hz}$ ) while under brief anesthesia, and another cohort received brief anesthesia alone ( $\sim 2 \%$ isoflurane for $\sim 8 \mathrm{~min}$, sham control). Passive visual stimulation significantly enhanced the recovery of spatial acuity in the previously deprived eye (visual stimulation, $0.652 \pm 0.038$ cycles/degree, $n=7$; sham controls, $0.390 \pm 0.076$ cycles/degree, $n=6$; Kruskal-Wallis test, $P=0.022$ ) (Fig. $4 \mathrm{~B}$ ). The recovery of visual acuity in the deprived eye was accompanied by an increase in the strength and selectivity of visually evoked neuronal responses.

Polar plots and post-stimulus time histograms of a representative layer IV neuron (recorded from deprived visual cortex) demonstrate the recovery of orientation selectivity of deprived eye visual responses (Fig. 4C,D). The cumulative distributions of all visually evoked activity confirm a significant increase in the strength (evoked activity [spikes/sec], deprived eye vs. deprived eye + visual stimulation + active visual discrimination, KS test, $P=0.029$ ) (Fig. 4E), and selectivity of visually evoked responses (KS test, $P=0.007$ ) (Fig. 4F). Thus passive visual stimulation and active visual discrimination can be used in combination to recover the visual deficits of severe amblyopia.

\section{Discussion}

Chronic monocular deprivation induces severe amblyopia, characterized by a significant decrease in the strength and selectivity of visual cortical responses. These deficits, combined with the decrease in synaptic plasticity that occurs over development, present significant challenges to recovery from severe amblyopia in adulthood. We have previously shown that dark exposure enhances synaptic plasticity in the adult visual cortex (He et al. 2006, 2007; Montey and Quinlan 2011). Here we show that passive viewing of simple visual stimuli can be used to rapidly and broadly strengthen visually evoked responses in the visual cortex of dark-exposed amblyopes. Importantly, passive visual stimulation significantly improved the recovery of spatial acuity driven by active visual discrimination in dark-exposed amblyopes. Together this demonstrates that significant opportunities exist for the use of visual stimulation to promote recovery from severe amblyopia in adulthood.

\section{The visual cortex of dark-exposed amblyopes is hyperplastic}

It is becoming increasingly apparent that visual responses can be regulated by passively viewing simple visual stimuli. In binocular mice, the enhancement of visually evoked responses by passive viewing of simple visual stimuli is typically slow and highly stimulus-selective (Sawtell et al. 2003; Frenkel et al. 2006, Cooke and Bear 2010). Interestingly, in dark-exposed amblyopes, passive visual stimulation induced an increase in visually evoked responses that was neither slow, nor stimulus-selective. Rapid and nonstimulus specific enhancement of visually evoked response was only observed in subjects that were chronically deprived and dark exposed. The reduced stimulus-selectivity induced by chronic monocular deprivation would allow a simple visual stimulus to produce more widespread, correlated neuronal activity in visual cortex. In addition, dark-exposure engages mechanisms that lower the threshold for synaptic plasticity in principle neurons (Cooper and Bear 2012), including the scaling up of AMPARs at excitatory synapses (Goel and Lee 2007) and the reactivation of endocannabinoid-dependent synaptic depression at inhibitory synapses (Huang et al. 2010). Dark exposure also increases the expression of NR2b-containing NMDARs, thereby enhancing the temporal summation of NMDAR-mediated synaptic currents and expanding the integration window for spike-timing dependent synaptic plasticity (Yashiro et al. 2005; He et al. 2006; Guo et al. 2012). Indeed, the combination of chronic monocular deprivation with dark exposure appears to render the adult visual cortex hyperplastic. This predicts that visual stimulation would be more effective at broadly strengthening visual responses in dark-exposed amblyopes than in binocular subjects. Interestingly, the gains in visual acuity promoted by active visual discrimination are less input-specific in human amblyopes than in nonamblyopic subjects (Huang et al. 2008; Astle et al. 2010). Importantly, dark-exposure enhances the experience-dependent recovery from 
A

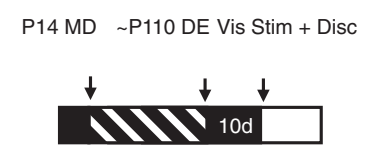

C

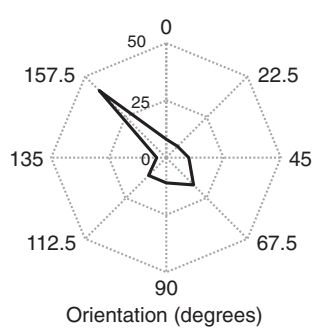

E

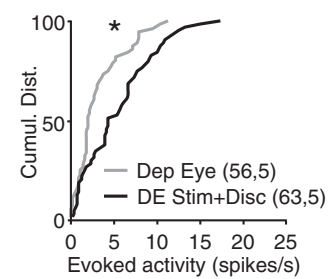

B

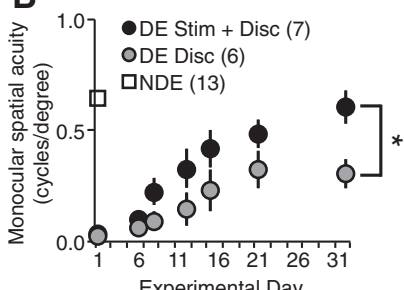

D Experimental Day
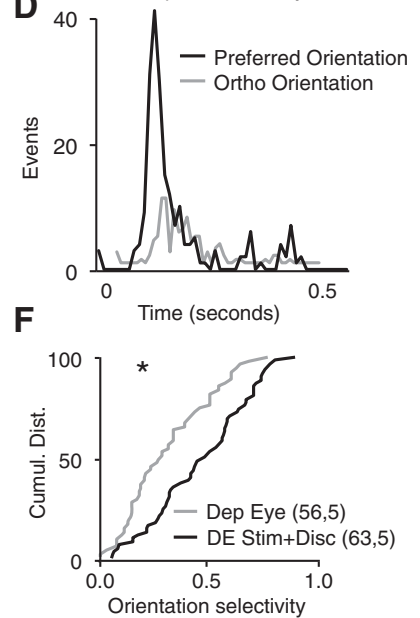

Figure 4. Passive visual stimulation improves the recovery of spatial acuity. $(A)$ Experimental timeline: chronically deprived subjects were trained to perform a visual discrimination task with the nondeprived eye. Following assessment of nondeprived eye spatial acuity, subjects received dark exposure (DE at $\sim P 110$ ), reverse deprivation, and began trials of active visual discriminations (Disc) with the chronically deprived eye. One cohort received passive visual stimulation prior to active visual discrimination (Vis Stim + Disc). (B) Passive visual stimulation followed by active visual discrimination enhances the recovery of spatial acuity (one-way ANOVA, $F_{(2,25)}=5.6914, P=0.010$, Stim only [black] versus Stim + Disc [gray]; [ $\left.{ }^{*}\right] P<0.05$, Tukey-Kramer HSD post hoc). (C) Polar plots of a representative layer IV neuron revealed an increase in the strength and orientation selectivity of visually evoked spiking output in the previously deprived eye. (D) Post-stimulus time histograms of spiking output of a representative layer IV neuron evoked by visual stimuli of preferred (black) and orthogonal (gray) orientations presented to previously deprived eye. $(E)$ The cumulative distribution of single unit activity revealed a significant increase in the strength of visually evoked spiking output in the previously deprived eye (Dep Eye [gray] 56,5, DE Stim + Disc [black]) 63,5, KS test, $P=0.029, n=$ units, subjects). ( $F$ ) The cumulative distribution of single unit activity reveals a significant increase in the orientation selectivity of neuronal spiking output in the previously deprived eye (DE [gray]) vs. DE Stim + Disc [black]), KS test, $P=0.007, n=$ units, subjects).

amblyopia in kittens, demonstrating that this paradigm can be successfully applied to animals with a columnar distribution of ocular dominance (Duffy and Mitchell 2013).

\section{Visual stimulation improves acuity}

The gains in spatial acuity observed during active visual discriminations are incremental in both humans and animal models. The daily incremental improvements may reflect that performance in the task induces a submaximal increase in synaptic strength that accumulates over days to reach a final plateau. In contrast, synaptic strengthening may be rapidly saturated, but continued training may extend the "ceiling" for subsequent synaptic plasticity (Rioult-Pedotti et al. 2007; Gagolewicz and Dringenberg 2011). Alternatively, a period of consolidation may be necessary to amplify changes to the level of detection, as has been proposed

for human visual perceptual learning (Karni and Sagi 1993). Nonetheless, visual discriminations alone can produce a modest recovery from severe amblyopia in rodents, which can be accelerated by dark-rearing (Iny et al. 2006; He et al. 2007). However, in the absence of visual stimulation, the recovery from severe amblyopia is incomplete.

\section{Experience-dependent changes persist in anesthetized subjects}

In our experiments, passive visual stimulation was presented to head-restrained subjects that were briefly anesthetized. The persistence of plasticity of visual responses in isoflurane was not surprising, given that neuronal response strength and selectivity are unchanged under isoflurane anesthesia (Villeneuve and Casanova 2003). In contrast, barbiturate anesthesia can mask experience-dependent synaptic plasticity in the adult rodent visual cortex (Pham et al. 2004), while ketamine-xylazine disrupts the consolidation of ocular dominance shifts induced by monocular deprivation in kittens (Rauschecker and Hahn 1987). The persistence of the enhancement of synaptic strength by visual stimulation under anesthesia demonstrates that attention/motivation are not required for this type of plasticity (Watanabe et al. 2001). However, it is important to note that passive visual stimulation did not promote a recovery of neuronal orientation selectivity or spatial acuity in dark-exposed amblyopes. The motivation and sustained visual attention necessary to perform the more complex spatial frequency discrimination task may be necessary to engage mechanisms of plasticity that drive the recovery of stimulus selectivity (Niell and Stryker 2010). Indeed, active visual experience, but not passive visual observation, has been shown to promote the emergence of orientation selectivity in the visual cortex of dark-reared kittens (Buisseret et al. 1978). A similar complexity is thought to explain the effectiveness of action video games over other types of visual stimulation in improving visual acuity in human amblyopes (Li et al. 2011).

The monocular spatial acuity that we report for the nondeprived eye of severe amblyopes is lower than in previous reports of binocular spatial acuity in adult rats (Prusky et al. 2000). Chronic monocular deprivation may compromise vision in the nondeprived eye, as reported in humans and felines (Giaschi et al. 1992; Chatzistefanou et al. 2005; Duffy and Mitchell 2013). In addition, acuities derived from repeated visual discriminations may contain a learned component. Indeed, challenging our subjects to perform that spatial frequency discrimination task with visual stimuli of novel orientations significantly reduced performance in the task, suggesting an experience-dependent and stimulus-selective enhancement of spatial acuity over baseline.

Although we limited our physiological recordings to primary visual cortex, the recovery of spatial acuity may require the enhancement of synaptic input, integration, and spiking output beyond primary visual cortex. However, the stimulus-specificity that is typical of the response enhancement induced by visual stimulation implicates contributions from synapses early in the visual cortical pathway, where neurons are selective for fundamental features of a visual stimulus (Dräger 1975; Karni and Sagi 1991; Niell and Stryker 2008). Indeed, both passive viewing and active discrimination modulate neuronal response properties in the primary visual cortex (Schoups et al. 2001; Schwartz et al. 2002; Teyler et al. 2005; Frenkel et al. 2006; Hua et al. 2010; Sale et al. 2011).

\section{Optimization of recovery from severe amblyopia}

Behavioral therapies aimed at stimulating the recovery from severe amblyopia in adulthood must address the decrease of 
neuronal response strength and selectivity induced by a chronic asymmetry in visual input, as well as the loss of synaptic plasticity that occurs over development. Passive visual stimulation induces a rapid and widespread increase in the strength of visually evoked responses, and enhances the experience-dependent recovery from spatial acuity in dark-exposed amblyopes. Passive visual stimulation may therefore be a simple, noninvasive adjunct to enhance existing visual discrimination paradigms to promote the recovery from severe amblyopia.

\section{Materials and Methods}

\section{Animal treatment}

\section{Subjects}

Long Evans rats (equal numbers of males and females) were raised on a cycle of 12-h light/12-h dark, with food and water available ad libitum. All procedures conformed to the guidelines of the US Department of Health and Human Services and the University of Maryland Institutional Animal Care and Use Committee. All efforts were made to minimize animal suffering and to reduce the number of animals used. A cohort received chronic monocular deprivation via eyelid suture from eye opening (about postnatal day 14) until adulthood (postnatal day P120 \pm 9.78 ). Eyelid suture was performed under ketamine/xylazine anesthesia $(50 \mathrm{mg} / 10$ $\mathrm{mg} / \mathrm{kg}$, i.p.), and subjects were disqualified in the event of suture opening or infection. Dark exposure (10 d beginning at $\sim \mathrm{P} 120$ ) was performed in a light-tight room under infrared illumination. Immediately following dark exposure, the chronically deprived eye was opened and the nondeprived eye closed (reverse deprivation, beginning at $\sim$ P130) prior to electrophysiological recordings and behavioral testing.

\section{Electrophysiology}

In vivo electrophysiology was performed under isoflurane anesthesia ( $\sim 2 \%$ in $100 \% \mathrm{O}_{2}$ delivered through the nose). The dura covering binocular visual cortex was exposed through a hole ( $\sim 3-\mathrm{mm}$ diameter) in the skull. Recordings were made from the binocular visual cortex (site of largest ipsilateral eye VEP, typically $7 \mathrm{~mm}$ posterior of bregma, $4 \mathrm{~mm}$ lateral of midline) with a tungsten microelectrode $(1 \mathrm{M} \Omega)$ relative to a ground screw in the frontal bone. VEPs (50-Hz low pass filter) were isolated from layer IV, and confirmed by polarity of the VEP waveform and latency to VEP peak. In passive viewing experiments, repetitive visual stimulation (100 cycles of 0.05 cycles/degree, 100\% contrast full-field vertical gratings reversing at $0.5 \mathrm{~Hz}$ ) was presented monocularly to the chronically deprived eye of amblyopes or the dominant, contralateral eye of binocular subjects. The average response to the first viewing of this stimulus was reported as baseline VEP ( 0 min), and the stimulus was repeated twice to collect VEPs 120 and $240 \mathrm{~min}$ later. Subjects were head fixed and anesthetized ( $\sim 2 \%$ isoflurane) during passive visual stimulation. After acquisition of the VEP at the third time point (240 min) with familiar vertical grating, VEPs were acquired in response to visual stimuli of novel orientations (seven orientations separated by 22.5 degrees) and spatial frequencies ( $0.08-1.2$ cycles/degree) to examine input specificity.

A $300-3-\mathrm{kHz}$ band pass filter was used to isolate multi-unit activity, which was sorted into single units based on waveform shape and principal component analysis (OpenEx software). The preferred orientation of the visual stimulus (from eight orientations) was determined for each unit after subtraction of the mean spontaneous activity (responses to blank screen in spikes/ $\mathrm{sec})$. Orientation selectivity index was calculated as (pref-ortho)/ (pref + ortho), where pref is the peak response (spikes/peak 10 msec bin) at the preferred orientation and ortho is the peak response at the orthogonal orientation. All neurons in which evoked activity $>$ spontaneous activity were included in the analyses.

\section{Spatial frequency discrimination task}

A two-alternative, forced-choice, water-based spatial frequency discrimination task, adapted from Prusky et al. 2000, was used to assess spatial acuity and promote recovery from amblyopia. Briefly, adults that received monocular deprivation at eye opening were trained with their nondeprived eye to associate a hidden escape platform (positive stimulus) with a high contrast (100\%) vertical sinusoidal grating ( 0.208 cycles/degree), and the absence of the escape platform (negative stimulus) with a gray computer screen of equal luminance $\left(75 \mathrm{~cd} / \mathrm{m}^{2}\right)$, white balance $(5000 \mathrm{~K})$ gamma value (1.8, calibrated with the Lacie Blue Eye Pro version 3.4). After familiarization with the task $(\sim 7 \mathrm{~d})$, a lane divider was introduced $(53.4 \mathrm{~cm})$ to define a discrimination point from which spatial acuity could be calculated. The location of the positive stimulus was moved from the left and right lanes in pseudorandom order. Subjects repeat trials to discriminate between 0.208 cycles/degree grating and gray screen until they perform $\geq 90 \%$ correct choices in three consecutive sessions of 10 trials (task learning criterion). To estimate spatial acuity, the spatial frequency of the positive visual stimulus was increased in 0.05 cycles/degree increments each time the subjects achieved $\geq 60 \%$ correct choices in a session of three to 10 trials. After performance plateaus with vertical gratings, the visual stimulus was changed to a novel orientation (horizontal gratings) and repeated. Chronically deprived subjects then received $10 \mathrm{~d}$ of dark exposure followed by reverse deprivation (open chronically deprived eye and close the nondeprived eye). The spatial frequency discrimination task was then repeated (beginning with 0.208 cycles/degree grating) to assess the spatial acuity of the deprived eye. One cohort received passive visual stimulation (100 cycles of 0.05 cycles/ degree, $100 \%$ contrast full-field vertical gratings reversing at 0.5 $\mathrm{Hz}$ ) while anesthetized with $2 \%$ isoflurane, and another cohort received brief $(\sim 8 \mathrm{~min})$ of isoflurane anesthesia alone each day that the visual discrimination task was performed. Spatial frequency discrimination trials were performed three times per week for 35 sessions, with an average of $\sim 25$ trials/day. Five subjects developed an opaque lens following reverse deprivation or a bias for one side during the visual discrimination task, and were disqualified.

\section{Statistics}

A one-way ANOVA, repeated measures ANOVA or a KruskalWallis test $(P<0.05)$ was used to determine the statistical significance between three or more independent experimental groups, followed by a post hoc analysis for pair-wise comparisons, when appropriate. A KS test was used to determine the statistical significance between the distributions of two independent data sets $(P<$ $0.05)$. A paired or unpaired $t$-test was used to determine statistical significance between two experimental groups $(P<0.05)$.

\section{Acknowledgments}

This work was supported by the National Institute on Aging F31AG034021 (K.L.M.) and the National Institutes of Health R01EY016431 (E.M.Q.).

\section{References}

Astle AT, Webb BS, McGraw PV. 2010. Spatial frequency discrimination learning in normal and developmentally impaired human vision. Vision Res 50: 2445-2454.

Beste C, Wascher E, Güntürkün O, Dinse HR. 2011. Improvement and impairment of visually guided behavior through LTP- and LTD-like exposure-based visual learning. Curr Biol 21: 876-882.

Buisseret P, Gary-Bobo E, Imvert M. 1978. Ocular motility and recovery of orientational properties of visual cortical neurones in dark-reared kittens. Nature 272: 816-817.

Chatzistefanou KI, Theodossiadis GP, Damanakis AG, Ladas ID, Moschos MN, Chimonidou E. 2005. Contrast sensitivity in amblyopia: The fellow eye of untreated and successfully treated amblyopes. J AAPOS 9: 468-474.

Cooke SF, Bear MF. 2010. Visual experience induces long-term potentiation in the primary visual cortex. J Neurosci 30: 16304-16313. 
Cooper LN, Bear MF. 2012. The BCM theory of synapse modification at 30: Interaction of theory with experiment. Nat Rev Neurosci 13: 798-810.

Dräger UC. 1975. Receptive fields of single cells and topography in mouse visual cortex. J Comp Neurol 160: 269-290.

Duffy KR, Mitchell DE. 2013. Darkness alters maturation of visual cortex and promotes fast recovery from monocular deprivation. Curr Biol 23: 382-386.

Frenkel MY, Sawtell NB, Diogo AC, Yoon B, Neve RL, Bear MF. 2006. Instructive effect of visual experience in mouse visual cortex. Neuron 51: $339-349$.

Gagolewicz PJ, Dringenberg HC. 2011. NR2B-subunit dependent facilitation of long-term potentiation in primary visual cortex following visual discrimination training of adult rats. Eur J Neurosci 34: $1222-1229$.

Giaschi DE, Regan D, Kraft SP, Hong XH. 1992. Defective processing of motion-defined form in the fellow eye of patients with unilateral amblyopia. Invest Ophthalmol Vis Sci 33: 2483-2489.

Giffin F, Mitchell DE. 1978. The rate of recovery of vision after early monocular deprivation in kittens. J Physiol 274: 511-537.

Goel A, Lee HK. 2007. Persistence of experience-induced homeostatic synaptic plasticity through adulthood in superficial layers of mouse visual cortex. J Neurosci 27: 6692-6700.

Guo Y, Huang S, de Pasquale R, McGehrin K, Lee HK, Zhao K, Kirkwood A 2012. Dark exposure extends the integration window for spike-timing-dependent plasticity. J Neurosci 32: 15027-15035.

He HY, Hodos W, Quinlan EM. 2006. Visual deprivation reactivates rapid ocular dominance plasticity in adult visual cortex. J Neurosci 26: $2951-2955$.

He HY, Ray B, Dennis K, Quinlan EM. 2007. Experience-dependent recovery of vision following chronic deprivation amblyopia. Nat Neurosci 10: $1134-1136$.

Hua T, Bao P, Huang CB, Wang Z, Xu J, Zhou Y, Lu ZL. 2010. Perceptual learning improves contrast sensitivity of V1 neurons in cats. Curr Biol 20: $887-894$.

Huang C, Zhou Y, Lu Z. 2008. Broad bandwidth of perceptual learning in the visual system of adults with anisometropic amblyopia. Proc Natl Acad Sci 105: 4068-4073.

Huang S, Gu Y, Quinlan EM, Kirkwood A. 2010. A refractory period for rejuvenating GABAergic synaptic transmission and ocular dominance plasticity with dark exposure. J Neurosci 30: 16636-16642.

Iny K, Heynen AJ, Sklar E, Bear MF. 2006. Bidirectional modifications of visual acuity induced by monocular deprivation in juvenile and adult rats. J Neurosci 26: 7368-7374.

Karni A, Sagi D. 1991. Where practice makes perfect in texture discrimination: Evidence for primary visual cortex plasticity. Proc Natl Acad Sci 88: 4966-4970.

Karni A, Sagi D. 1993. The time course of learning a visual skill. Nature 365: $250-252$.

Lennerstrand G, Kvarnström G, Lundh BL, Wranne K. 1981. Effects of grating stimulation on visual acuity in amblyopia. Acta Ophthalmol (Copenh) 59: 179-188.

Levi DM, Li RW. 2009. Perceptual learning as a potential treatment for amblyopia: A mini-review. Vision Res 49: 2535-2549.

Li RW, Ngo C, Nguyen J, Levi DM. 2011. Video-game play induces plasticity in the visual system of adults with amblyopia. PLoS Biol 9: e1001135.
Liao DS, Krahe TE, Prusky GT, Medina AE, Ramoa AS. 2004. Recovery of cortical binocularity and orientation selectivity after the critical period of ocular dominance plasticity. J Neurophysiol 92: 2113-2121.

Mitchell DE, MacKinnon S. 2002. The present and potential impact of research on animal models for clinical treatment of stimulus deprivation amblyopia. Clin Exp Optom 85: 5-18.

Montey KL, Quinlan EM. 2011. Recovery from chronic monocular deprivation following reactivation of thalamocortical plasticity by dark exposure. Nat Commun 2: 317.

Niell CM, Stryker MP. 2008. Highly selective receptive fields in mouse visual cortex. J Neurosci 28: 7520-7536.

Niell CM, Stryker MP. 2010. Modulation of visual response by behavioral state in mouse visual cortex. Neuron 65: 472-479.

Pham TA, Graham SJ, Suzuki S, Barco A, Kandel ER, Gordon B, Lickey ME. 2004. A semi-persistent adult ocular dominance plasticity in visual cortex is stabilized by activated CREB. Learn Mem 11: 738-747.

Prusky GT, West PWR, Douglas RM. 2000. Behavioral assessment of visual acuity in mice and rats. Vision Res 40: 2201-2209.

Rauschecker JP, Hahn S. 1987. Ketamine-xylazine anesthesia blocks consolidation of ocular dominance changes in kitten visual cortex. Nature 326: 183-185.

Rioult-Pedotti MS, Donoghue JP, Dunaevsky A. 2007. Plasticity of the synaptic modification range. J Neurophysiol 98: 3688-3695.

Sale A, De Pasquale R, Bonaccorsi J, Pietra G, Olivieri D, Berardi N, Maffei L. 2011. Visual perceptual learning induces long-term potentiation in the visual cortex. Neuroscience 172: 219-225.

Sawtell NB, Frenkel MY, Philpot BD, Nakazawa K, Tonegawa S, Bear MF. 2003. NMDA receptor-dependent ocular dominance plasticity in adult visual cortex. Neuron 38: 977-985

Schoups A, Vogels R, Qian N, Orban G. 2001. Practicing orientation identification improves orientation coding in V1 neurons. Nature 412: $549-553$.

Schwartz S, Maquet P, Frith C. 2002. Neural correlates of perceptual learning: A functional MRI study of visual texture discrimination. Proc Natl Acad Sci 99: 17137-17142.

Teyler TJ, Hamm JP, Clapp WC, Johnson BW, Corballis MC, Kirk IJ. 2005. Long-term potentiation of human visual evoked responses. Eur $J$ Neurosci 21: 2045-2050.

Villeneuve MY, Casanova C. 2003. On the use of isoflurane versus halothane in the study of visual response properties on single cells in the primary visual cortex. J Neurosci Methods 129: 19-31.

Watanabe T, Náñez JE, Sasaki Y. 2001. Perceptual learning without perception. Nature 413: $844-848$.

Wiesel TN, Hubel DH. 1965. Extent of recovery from the effects of visual deprivation in kittens. J Neurophysiol 28: 1060-1072.

Willshaw HE, Malmheden A, Clarke J, Williams A, Dean L. 1980 Experience with the CAM vision stimulator: Preliminary report. $\mathrm{Br} J$ Ophthalmol 64: 339-341.

Yashiro K, Corlew R, Philpot BD. 2005. Visual deprivation modifies both presynaptic glutamate release and the composition of perisynaptic/ extrasynaptic NMDA receptors in the adult visual cortex. J Neurosci 25: $11684-11692$.

Zhou Y, Huang C, Xu P, Tao L, Qiu Z, Li X. 2006. Perceptual learning improves contrast sensitivity and visual acuity in adults with anisometropic amblyopia. Vision Res 46: 739-750.

Received January 14, 2013; accepted in revised form March 19, 2013. 


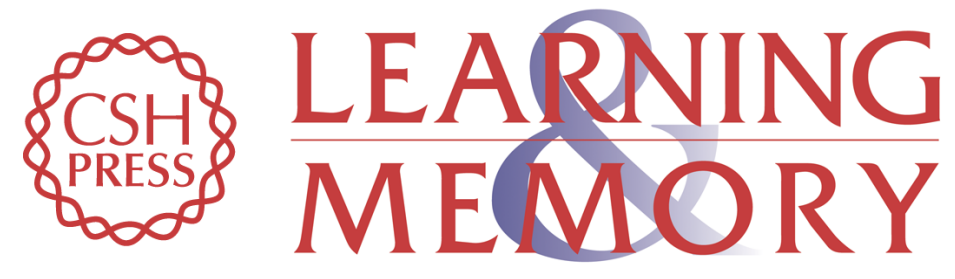

\section{Repetitive visual stimulation enhances recovery from severe amblyopia}

Karen L. Montey, Nicolette C. Eaton and Elizabeth M. Quinlan

Learn. Mem. 2013, 20:

Access the most recent version at doi:10.1101/Im.030361.113

References This article cites 45 articles, 14 of which can be accessed free at: http://learnmem.cshlp.org/content/20/6/311.full.html\#ref-list-1

License

Email Alerting Receive free email alerts when new articles cite this article - sign up in the box at the Service top right corner of the article or click here. 